\title{
Correlation of serum high-sensitivity C-reactive protein and interleukin-6 in patients with acute coronary syndrome
}

\author{
X.H. Wang, S.Q. Liu, Y.L. Wang and Y. Jin \\ Department of Cardiology, Liaocheng People's Hospital, Liaocheng, \\ Shandong, China \\ Corresponding author: X.H. Wang \\ E-mail: xiaohuawangcn@163.com
}

Genet. Mol. Res. 13 (2): 4260-4266 (2014)

Received May 14, 2013

Accepted September 13, 2013

Published June 9, 2014

DOI http://dx.doi.org/10.4238/2014.June.9.11

\begin{abstract}
Serum high-sensitivity C-reactive protein (hs-CRP) is a sensitive indicator of inflammation, which is closely related with the progress of plaque formation. Interleukin-6 (IL-6) is one of the inflammatory markers of local coronary plaque and the peripheral blood cycle, promoting the occurrence of atherosclerosis development and plaque rupture. In this study, the correlation of hs-CRP and IL-6 was investigated in patients with acute coronary syndrome (ACS). Sixty cases of ACS, including 33 cases of acute myocardial infarction (AMI) and 27 cases of unstable angina pectoris (UAP), 45 cases of stable angina pectoris (SAP), and 45 healthy people (HG) were enrolled in study. The serum hs-CRP and serum IL-6 levels were tested by the immune turbidimetric method and enzyme-linked immunosorbent assay (ELISA), respectively. The differences among groups and their correlations were evaluated. Results showed that the serum hs-CRP and IL- 6 concentrations of the AMI and UAP groups were significantly higher than those of the SAP and HG groups, respectively $(\mathrm{P}<0.01)$, and those of the AMI group were significantly higher than those of the UAP group $(\mathrm{P}<0.05)$. The serum hs-CRP and IL-6 levels of the ACS
\end{abstract}


group were positively correlated $(r=0.836)$. The serum hs-CRP and IL-6 levels could be used to determine the stability of plaque, and have some relevance in the ACS process, showing great value in judgments of ACS prognosis.

Key words: Acute coronary syndrome; Interleukin-6; Correlation; High-sensitivity C-reactive protein

\section{INTRODUCTION}

Acute coronary syndrome (ACS) can cause malignant cardiovascular events such as sudden cardiac death, and its occurrence is mainly due to the collapsing loss, fracturing, and rupturing of coronary plaque, thrombosis, and other factors. The instability of plaque forms the pathological basis of ACS (Plutzky, 2001). Therefore, identification of unstable plaques can help to screen ACS patients to carry out early interventions, which can reduce or avoid the occurrence of adverse cardiovascular events.

Intravascular ultrasound and vascular endoscopy are two invasive examination methods that could be used to identify unstable plaque (Naghavi et al., 2003). Multi-slice computed tomography (CT), as a noninvasive method, is now increasingly used in clinical coronary heart disease diagnosis for distinguishing soft plaque from hard plaque through the contrast of gray values. However, these means not only require expensive equipment, but also are currently too expensive to be popularized. Searching for a non-invasive mean that could generally be carried out has become a broad internal clinical demand.

Inflammation is always present throughout the period of ACS, and its degree of activity is closely related to the instability of plaque. Serum high-sensitivity C-reactive protein (hs-CRP) is a sensitive indicator of inflammation, which is closely related with the progress of plaque. Hs-CRP levels are significantly higher in ACS patients with unstable plaque (thin fibrous cap) (Kashiwagi et al., 2009), and such patients usually show poor prognosis (Nakachi et al., 2008). Therefore, hs-CRP levels can reflect the prognosis of ACS patients; however, hs-CRP is a non-specific inflammatory factor, and therefore has poor specificity.

Interleukin-6 (IL-6) is considered one of the inflammatory markers of local coronary plaque and the peripheral blood cycle, and can be detected with high concentrations in lipid stripe pure fiber blocks of atherosclerotic plaque (Rus et al., 1996). IL-6 can promote the occurrence of atherosclerosis development and plaque rupture (Ikeda et al., 2001). When plaque has inflammation or rupturing, the blood concentrations of IL-6 tend to increase. IL-6 and other cytokines were significantly increased in ACS patients (De Gennaro et al., 2012). Lai et al. (2011) also found that serum IL-6 levels of plaque in ACS patients were significantly higher than those of the control group, while IL-6 levels of the unstable and mixed plaque group were also significantly higher than the control group. In addition, Burazor et al. (2008) found that IL-6 levels in ACS patients were higher than those of healthy controls. Other studies have shown that the IL-6 plasma concentration in ACS patients was related to the risk of serious coronary events (Mazzone et al., 1999; Ridker et al., 2000). However, the relationship between IL-6 and ACS is not consistent across other studies. One study showed that IL-6 levels in patients with unstable angina were significantly higher than those in patients with ACS 
(102.4 \pm 1.9 vs 224.6 $\pm 3.6, \mathrm{P}=0.007)$ (Sarrafzadegan et al., 2012). Therefore, the relationship between IL-6 and ACS needs further clarification.

Some studies have suggested that hs-CRP and IL-6 may reflect the intensity of plaque inflammation and predict plaque brittleness (Plutzky, 2001). Therefore, whether the joint determination of hs-CRP and IL-6 levels can better assess the prognosis of ACS than single determinations of each element remains to be elucidated. In this study, 60 ACS patients were tested for serum inflammatory markers, namely hs-CRP and IL-6, and the data were compared with those of stable angina pectoris (SAP) patients and healthy people (HG). The correlations within and among groups were analyzed. The objective of this study was to determine whether hs-CRP and IL-6 could be used as serum reference indexes for evaluations of plaque block instability in the ACS process.

\section{MATERIAL AND METHODS}

\section{Research subjects}

Sixty ACS patients who were treated in our hospital's cardiac medical ward between January 2010 and December 2011 were selected for this study. Their diagnoses were all in line with the standards of the Chinese Medical Society of Cardiology in 2000 and 2001. Among these patients, 33 cases were acute myocardial infarction (AMI) (27 males and 6 females, aged 61.5-86.0 years, average $66.0 \pm 3.4$ years), 27 cases were unstable angina pectoris (UAP) (22 males and 5 females, aged 61.4-78.2 years, average $67.3 \pm 2.1$ years), and another 45 cases were SAP ( 38 males and 7 females, aged 60.2-81.5 years, average $66.5 \pm 2.5$ years). Fortyfive elderly healthy people were randomly chosen as the healthy control group (HG), including 36 males and 9 females, aged 60.4-82.8 years (average $65.3 \pm 2.6$ years). The HG group did not show any coronary heart disease history, had normal echocardiograms (ECGs), and had negative exercise stress ECGs based on physical examination and laboratory tests. The exclusion criteria were as follows: progressive renal insufficiency, hepatic dysfunction, acute and chronic infection, heart dysfunction, surgery and trauma within 3 months, inflammation, anemia, peripheral vascular disease, suspected systemic thrombotic diseases and pregnancy, diabetes and cancer, other heart disease, thyroid dysfunction, and autoimmune diseases.

\section{Experimental methods}

Three milliliters fasting venous blood were collected the morning after enrollment in the hospital, and within $30 \mathrm{~min}$, all blood samples were centrifuged at $4{ }^{\circ} \mathrm{C}$ and at $3000 \mathrm{rpm}$ for $10 \mathrm{~min}$. The serum was separated, and stored at $-70^{\circ} \mathrm{C}$ for detection within 6 months. Hs-CRP and IL- 6 concentrations were determined after the specimen collection, with the simultaneous detection of lipids, blood glucose, and other related indicators. hs-CRP and IL-6 were divided into 3 batches for the detection in order to avoid batch and measurement errors, and doublebarreled duplicate detection was carried out. Serum hs-CRP levels were measured with the particle-enhanced immune turbidimetric method (Beckman) using the RIA analyzer. Serum IL-6 concentrations were detected using an enzyme-linked immunosorbent assay (ELISA) kit (provided by the Technology Development Center of the People's Liberation Army General Hospital, Beijing, China). 


\section{Statistical analysis}

Measurement data are reported as means $\pm \mathrm{SDs}$, and the count data are expressed as percentages. The measurement data within groups were compared using the Student's $t$-test or analysis of variance; the $\chi^{2}$ test was used to compare the count data. Spearman rank correlation analysis was used to evaluate correlations. All data were analyzed using the SPSS13.0 software.

\section{RESULTS}

\section{General information}

As shown in Table 1, the age, gender, body mass index (BMI), hypertension, diabetes, cholesterol, and other general indicators showed no significant differences among the different groups $(\mathrm{P}>0.05)$.

\begin{tabular}{|c|c|c|c|c|c|c|c|c|c|c|}
\hline Group & $\mathrm{N}$ & Age & $\mathrm{M} / \mathrm{F}$ & BMI $\left(\mathrm{kg} / \mathrm{m}^{2}\right)$ & $\begin{array}{c}\text { Hypertension } \\
\mathrm{N}(\%)\end{array}$ & $\begin{array}{c}\text { Diabetes } \\
\mathrm{N}(\%)\end{array}$ & $\begin{array}{c}\mathrm{TC} \\
(\mathrm{mM})\end{array}$ & $\begin{array}{c}\mathrm{TG} \\
(\mathrm{mM})\end{array}$ & $\begin{array}{l}\text { LDL-C } \\
(\mathrm{mM})\end{array}$ & $\begin{array}{c}\text { HDL-C } \\
(\mathrm{mM})\end{array}$ \\
\hline HG & 45 & $65.3 \pm 2.6$ & $36 / 9$ & $22.32 \pm 4.30$ & $5(11.1)$ & $3(6.8)$ & $5.2 \pm 1.6$ & $1.8 \pm 0.7$ & $2.8 \pm 0.8$ & $1.0 \pm 0.4$ \\
\hline SAP & 45 & $66.5 \pm 2.5$ & $38 / 7$ & $25.90 \pm 2.61$ & $6(13.6)$ & $6(13.6)$ & $6.0 \pm 1.5$ & $1.7 \pm 0.5$ & $3.0 \pm 1.1$ & $1.1 \pm 0.5$ \\
\hline UAP & 27 & $67.3 \pm 2.1$ & $22 / 5$ & $26.20 \pm 3.84$ & $4(16.5)$ & $4(16.5)$ & $5.7 \pm 1.2$ & $2.0 \pm 0.6$ & $2.8 \pm 1.1$ & $1.0 \pm 0.6$ \\
\hline AMI & 33 & $66.0 \pm 3.4$ & $27 / 6$ & $25.39 \pm 4.11$ & $6(18.3)$ & $7(22.4)$ & $6.3 \pm 1.0$ & $2.1 \pm 0.8$ & $3.1 \pm 1.1$ & $1.0 \pm 0.5$ \\
\hline
\end{tabular}

$\mathrm{BMI}=$ body mass index; $\mathrm{TC}=$ total cholesterol; $\mathrm{TG}=$ triglycerides; LDL-C $=$ low density lipoprotein; HDL-C = high density lipoprotein; $\mathrm{HG}=$ healthy control group; $\mathrm{SAP}=$ stable angina pectoris; $\mathrm{UAP}=$ unstable angina pectoris; AMI = acute myocardial infarction.

\section{Changes of hs-CRP and IL-6 concentrations in each group}

As shown in Table 2, hs-CRP and IL-6 concentrations in the AMI and UAP groups were significantly higher than those in the SAP and HG groups $(\mathrm{P}<0.05)$. Furthermore, the hs-CRP and IL-6 concentrations of the AMI group were significantly higher than those of the UAP group $(\mathrm{P}<0.05)$; hs-CRP and IL-6 concentrations did not differ significantly between the SAP and HG groups $(\mathrm{P}>0.05)$. In ACS patients (AMI + UAP), the serum hs-CRP and IL-6 levels were positively correlated with each other $(r=0.836)$.

\begin{tabular}{|c|c|c|c|}
\hline Group & $\mathrm{N}$ & hs-CRP (mg/L) & IL-6 (pg/mL) \\
\hline$\overline{\mathrm{HG}}$ & 45 & $1.35 \pm 1.03$ & $8.15 \pm 6.39$ \\
\hline SAP & 45 & $2.00 \pm 1.46$ & $10.70 \pm 8.10$ \\
\hline UAP & 27 & $5.95 \pm 1.60^{*} \geq$ & $24.41 \pm 8.68^{*}=$ \\
\hline AMI & 33 & $8.91 \pm 2.12^{*} \geq \mathbf{\Delta}$ & $32.50 \pm 9.32^{*}=\Delta$ \\
\hline
\end{tabular}

\section{DISCUSSION}

ACS, including UAP, AMI, and sudden cardiac death, is mainly caused by the cracks, 
ulcers, or rupture of unstable coronary atherosclerotic plaque, which could trigger thrombus formation (Nakachi et al., 2008). Previous studies have shown that $60-70 \%$ of ACS cases show only mild or moderate coronary stenosis, which is directly caused by coronary thrombosis of plaque rupture and the infiltration of local inflammatory cells; furthermore, systemic inflammation is the main cause of plaque rupture (Biasucci et al., 1999). Hs-CRP and IL-6 are sensitive markers of inflammation, which are commonly used in the detection of mild inflammatory reactions. In this study, 33 patients with AMI and 27 patients with UAP were studied, and the results showed that the hs-CRP and IL- 6 concentrations of the ACS group were significantly higher than those of the SAP and HG groups, indicating that inflammation played an important role in the pathological process of ACS.

Dynamic changes of plaque rupture and mural thrombus are the pathological basis of the vast majority of ACS cases. There is no positive correlation between plaque rupture and the severity of coronary artery stenosis, whose risk is mainly determined by plaque type and external factors rolling on the plaque (Rabbani and Topol, 1999), and the type of plaque is the key factor for determining its stability. These easily ruptured plaques contain a large lipid-rich core, and the weak areas can be seen on the fibrous cap collagen fibers. In addition, vascular smooth muscle cells are reduced in this region, often locating in the junction between the fibrous cap and intima (shoulder region). Plaque contains a large number of activated macrophages and foam cells, and the content of activated $\mathrm{T}$ cells and mast cells increases, while the content of vascular smooth muscle cells is reduced. This indicates that the inflammatory response plays an important role in plaque rupture.

Hs-CRP is a chemotactic factor inside fibrinogen, and fibrinogen could make macrophages adhere to the endothelial surface, which would then migrate into the intima, thus promoting plaque rupture and vasoconstriction. Therefore, the elevation of hs-CRP levels in ACS, as instability markers of atherosclerotic lesions, can prompt the coronary plaque instability. There is a large amount of hs-CRP deposition in human early coronary plaque, and the binding of hs-CRP and lipoprotein could activate the complementary system via the classical pathway, resulting in a large number of terminal attack complexes, leading to intimal injury and even plaque rupture. In addition, hs-CRP could promote monocytes to release tissue factors, which would initiate the extrinsic coagulation system and promote thrombosis (Beaudeux et al., 2004). At the same time, hs-CRP is a sensitive indicator of the body's inflammatory response, and its blood level begins to increase after being stimulated for $6 \mathrm{~h}$. The increase extent is almost perfectly positively proportional to the extent of inflammation and tissue damage. As a new risk factor, hs-CRP could be viewed as an independent predictor of very early and long-term death of revascularization-postoperative in non-ST-segment elevation patients (Rus and Niculescu, 1997). Hs-CRP levels would be expected to be significantly higher in the presence of inflammation, and recent studies have shown that even a very small increase in hs-CRP could accurately indicate the presence of inflammation. In this study, the hs-CRP level was higher in the ACS group compared to the SAP and HG groups, and was particularly high in the AMI group, whereas there was no statistically significant difference between the SAP and HG groups.

IL-6 might be involved in the occurrence and development of ACS through a variety of mechanisms, such as by promoting macrophages to express cell adhesion molecules, matrix metalloproteinases, and tissue factors, inducing hepatocytes to synthesize fiber fibrinogen and hs-CRP, as well as by promoting platelet aggregation and vascular smooth muscle cell prolif- 
eration (Mueller et al., 2002).

Basic research revealed that IL-6 and hs-CRP might participate in ACS with the following three mechanisms. First, IL-6 might promote inflammatory effector cells to express the adhesion-associated proteins, chemokines, and then promote inflammatory cell adhesion and aggregation to form plaque. This would promote the activity of inflammatory cells to increase the generation of oxygen free radicals and increase matrix metalloproteinase expression, thus leading to plaque instability (Verma et al., 2002). Second, IL-6 might activate the complement, thereby affecting the generation of nitric oxide and endothelin-1 (ET-1), leading to endothelial dysfunction (Blake and Ridker, 2002). Finally, IL-6 might promote the expression of the tissue factor type I plasminogen activator inhibition factor and enhance their activities, leading to clotting disorders and thrombus formation (Pasceri et al., 2000).

Cells producing IL-6 are present in the lesion sites of elderly ACS patients, which play a role in promoting the ACS process. In this study, compared with the HG group, hs-CRP and IL-6 levels were significantly increased in the AMI and UAP groups. Although these levels were higher in the SAP group compared to the HG group, the differences were not statistically significant. Furthermore, the results showed that there was a positive correlation between hs-CRP and IL-6, and they both increased in the serum of ACS patients. IL-6 stimulates liver cells to synthesize hs-CRP in a dose-dependent manner, and could stimulate the activated macrophages to produce hs-CRP. In addition, the blood hs-CRP level also has an impact on IL-6, and it is considered an IL-6 receptor agonist, facilitating the binding of IL-6 with the IL-6 receptor and allowing the formation of an IL-6 receptor/IL-6 complex. Peripheral blood plasma IL-6 levels are significantly higher, and the higher the IL-6 plasma concentration, the higher the risk of serious coronary events, with worse thrombolytic effects and poorer clinical prognosis (Koukkunen et al., 2001).

In conclusion, inflammation and extracellular matrix degradation play important roles in the pathogenesis of ACS. The serum hs-CRP and IL-6 levels can be used to determine the stability of plaque, and have some relevance in the ACS process, showing great value for judgments in ACS prognosis.

\section{REFERENCES}

Beaudeux JL, Giral P, Bruckert E, Foglietti MJ, et al. (2004). Matrix metalloproteinases, inflammation and atherosclerosis: therapeutic perspectives. Clin. Chem. Lab. Med. 42: 121-131.

Biasucci LM, Liuzzo G, Grillo RL, Caligiuri G, et al. (1999). Elevated levels of C-reactive protein at discharge in patients with unstable angina predict recurrent instability. Circulation 99: 855-860.

Blake GJ and Ridker PM (2002). C-reactive protein, subclinical atherosclerosis, and risk of cardiovascular events. Arterioscler. Thromb. Vasc. Biol. 22: 1512-1513.

Burazor I, Vojdani A and Burazor M (2008). Interrelationship of interleukin 6, C-reactive protein and Chlamydia pneumoniae IgG antibodies in patients with acute coronary syndromes. Vojnosanit. Pregl. 65: 425-433.

De Gennaro L, Brunetti ND, Montrone D, De Rosa F, et al. (2012). Subacute inflammatory activation in subjects with acute coronary syndrome and left ventricular dysfunction. Inflammation 35: 363-370.

Ikeda U, Ito T and Shimada K (2001). Interleukin-6 and acute coronary syndrome. Clin. Cardiol. 24: 701-704.

Kashiwagi M, Tanaka A, Kitabata H, Tsujioka H, et al. (2009). Relationship between coronary arterial remodeling, fibrous cap thickness and high-sensitivity C-reactive protein levels in patients with acute coronary syndrome. Circ. J. 73: 1291-1295.

Koukkunen H, Penttila K, Kemppainen A, Halinen M, et al. (2001). C-reactive protein, fibrinogen, interleukin-6 and tumour necrosis factor-alpha in the prognostic classification of unstable angina pectoris. Ann. Med. 33: 37-47.

Lai CL, Ji YR, Liu XH, Xing JP, et al. (2011). Relationship between coronary atherosclerosis plaque characteristics and high sensitivity C-reactive proteins, interleukin-6. Chin. Med. J. (Engl.) 124: 2452-2456.

Genetics and Molecular Research 13 (2): 4260-4266 (2014)

CFUNPEC-RP www.funpecrp.com.br 
Mazzone A, De SS, Vezzoli M, Fossati G, et al. (1999). Plasma levels of interleukin 2, 6, 10 and phenotypic characterization of circulating T lymphocytes in ischemic heart disease. Atherosclerosis 145: 369-374.

Mueller C, Buettner HJ, Hodgson JM, Marsch S, et al. (2002). Inflammation and long-term mortality after non-ST elevation acute coronary syndrome treated with a very early invasive strategy in 1042 consecutive patients. Circulation 105: 1412-1415.

Naghavi M, Libby P, Falk E, Casscells SW, et al. (2003). From vulnerable plaque to vulnerable patient: a call for new definitions and risk assessment strategies: Part I. Circulation 108: 1664-1672.

Nakachi T, Kosuge M, Hibi K, Ebina T, et al. (2008). C-reactive protein elevation and rapid angiographic progression of nonculprit lesion in patients with non-ST-segment elevation acute coronary syndrome. Circ. J. 72: 1953-1959.

Pasceri V, Willerson JT and Yeh ET (2000). Direct proinflammatory effect of C-reactive protein on human endothelial cells. Circulation 102: 2165-2168.

Plutzky J (2001). Inflammatory pathways in atherosclerosis and acute coronary syndromes. Am. J. Cardiol. 88: 10K-15K. Rabbani R and Topol EJ (1999). Strategies to achieve coronary arterial plaque stabilization. Cardiovasc. Res. 41: 402-417.

Ridker PM, Rifai N, Stampfer MJ and Hennekens CH (2000). Plasma concentration of interleukin-6 and the risk of future myocardial infarction among apparently healthy men. Circulation 101: 1767-1772.

Rus H, Vlaicu R and Niculescu F (1996). Interleukin-6 and interleukin-8 protein and gene expression in human arterial atherosclerostic all. Atherosclerosis 127: 263-271.

Rus H and Niculescu FI (1997). Inflammation, aspirin, and the risk of cardiovascular disease. N. Engl. J. Med. 337: 423424.

Sarrafzadegan N, Sadeghi M, Ghaffarpasand F, Alisaeidi A, et al. (2012). Interleukin-6 and E-selectin in acute coronary syndromes and stable angina pectoris. A comparative study. Herz 37: 926-930.

Verma S, Li SH, Badiwala MV, Weisel RD, et al. (2002). Endothelin antagonism and interleukin-6 inhibition attenuate the proatherogenic effects of C-reactive protein. Circulation 105: 1890-1896. 